\title{
Cataracts are Associated with the Coexistence of Moderate to Severe Obstructive Sleep Apnea and Diabetes Mellitus
}

\author{
Seungku Lee, $\mathrm{PhD}^{1 *}$, Dae Wui Yoon, $\mathrm{PhD}^{2 *}$, Jinkwan Kim, $\mathrm{PhD}^{2}$, Young-Woo Suh, MD, PhD³, Chol Shin, MD, PhD ${ }^{1,4}$ \\ IInstitute of Human Genomic Study, Korea University Ansan Hospital, Korea University, Ansan, Korea \\ 2Department of Biomedical Laboratory Science, College of Health Science, Jungwon University, Geosan, Korea \\ ${ }^{3}$ Division of Ophthalmology, Korea University Ansan Hospital, Ansan, Korea \\ ${ }^{4}$ Division of Pulmonary, Sleep, and Critical Care Medicine, Department of Internal Medicine, Korea University Ansan Hospital, Ansan, Korea
}

\author{
Received: April 20, 2020 \\ Revised: June 26, 2020 \\ Accepted: August 2, 2020 \\ Correspondence \\ Chol Shin, MD, PhD \\ Division of Pulmonary, \\ Sleep, and Critical Care Medicine, \\ Department of Internal Medicine, \\ Korea University Ansan Hospital \\ and Institute of Human Genomic Study, \\ Korea University, 123 Jeokgeum-ro, \\ Danwon-gu, Ansan 15355, Korea \\ Tel +82-31-412-5603 \\ Fax +82-31-412-5604 \\ E-mail chol-shin@korea.ac.kr \\ *These authors contributed equally to this work.
}

ORCID

Seungku Lee

https://orcid.org/0000-0001-5120-5557

Dae Wui Yoon

https://orcid.org/0000-0001-8875-2255

Jinkwan Kim

https://orcid.org/0000-0002-7123-1354

Young-Woo Suh

https://orcid.org/0000-0003-4857-206X

Chol Shin

https://orcid.org/0000-0002-2928-8576

(c) This is an Open Access article distributed under the terms of the Creative Commons Attribution Non-Commercial License (https://creativecommons.org/licenses/by-nc/4.0) which permits unrestricted non-commercial use, distribution, and reproduction in any medium, provided the original work is properly cited.
Background and Objective The individual presence of diabetes mellitus (DM) or obstructive sleep apnea (OSA) is significantly associated with cataract formation, but few studies have examined the association of cataracts with comorbid DM and OSA. Our aim in this study was to confirm the relationship between cataracts and the individual presence of DM or OSA in a large population-based cohort study and evaluate the association between cataracts and the coexistence of OSA and DM.

Methods We included 699 individuals who were enrolled in the Korean Genome and Epidemiology Study and who underwent both nocturnal polysomnography and cataract diagnosis in the study. We assessed the presence and severity of OSA by means of unattended home sleep monitoring. DM was diagnosed based on the fasting blood-glucose level or by the use of antihyperglycemic medications. Cataracts were diagnosed according to the Lens Opacities Classification System III.

Results The prevalence of cataracts tended to increase as OSA worsened. Diabetes patients had a higher prevalence of cataracts than did normal subjects. In multivariate analysis, there was no significant association of cataracts with DM or OSA alone. In the joint analysis of DM and OSA, however, the odds for cataracts were significantly higher in the DM patients with moderate to severe OSA than in the DM patients without OSA, indicating a significant synergy of moderate to severe OSA and DM on cataracts.

Conclusions The concurrent presence of DM and moderate to severe OSA was associated with cataracts. Thus, OSA should be considered in the prevention or treatment of diabetic cataracts.

Sleep Med Res 2020;11(2):129-134

Key Words Obstructive sleep apnea, Cataracts, Diabetes mellitus, Interaction.

\section{INTRODUCTION}

Obstructive sleep apnea (OSA) is a common respiratory symptom affecting $2-4 \%$ of the adult population worldwide [1]. OSA is characterized by repeated episodes of partial or complete pharyngeal obstruction during sleep. Such events accompany intermittent hypoxia and arousal, which increase oxidative stress, systemic inflammation, and sympathetic nervous-system surges. It is well known that these abnormal biochemical and physiological disturbances are key mechanisms for the pathogenesis of several chronic systemic diseases seen in patients with OSA, such as cardiovascular diseases and diabetes mellitus (DM) [2,3]. In addition to these systemic diseases, OSA is associated with local complications, including eye disorders. Associations of eye disorders, including glaucoma, floppy eyelid syndrome (FES), non-arteritic anterior ischemic optic neuropathy (NAION), keratoconus, diabetic retinopathy, visual field defects, with OSA have been reported [4-7]. According to a large meta-analysis, OSA was significantly as- 
sociated with glaucoma [4,5] and NAION [6]. OSA was not associated with diabetic retinopathy, but some evidence has suggested a significant association of OSA with greater severity of diabetic retinopathy and advanced diabetic retinopathy in type 2 DM patients [7]. Continuous positive airway pressure treatment of OSA improved the symptoms of FES [8] and visual field loss [9] but did not prevent the development of NAION in patients with sleep apnea syndrome [10].

Cataracts are a main cause of blindness and visual defects worldwide. The prevalence of cataracts increases with age. Large population-based studies have reported the prevalence to be from $3.9 \%$ at $55-64$ years to $92.6 \%$ at more than 80 years [11-13]. Cataract formation is a multifactorial process. Its risk factors include increasing age, female sex, genetic factors (e.g., chromosome 3 in KCNAB1 and chromosome 21 in CRYAA), lifestyle factors (e.g., ultraviolet-B exposure, cigarette smoking, and alcohol consumption), consumption of carbohydrates with a high glycemic index, and systemic and medical problems (e.g., type $2 \mathrm{DM}$, high systemic blood pressure, and metabolic syndrome) [14]. Although few studies have reported the association of OSA with cataracts, a recent cross-sectional study reported the magnitude and risk factors of ocular complications in OSA patients associated with a greater risk of senile cataracts [15]. However, to our knowledge, no study has investigated the association between OSA and cataracts in a large population-based prospective cohort study. Moreover, many studies have identified associations between cataracts and DM, but no study has examined the association between comorbid OSA and DM and the risk of cataracts. Therefore, we did this study to confirm the relationship between cataracts and the individual presence of DM or OSA in a large population-based cohort study. We also aimed to evaluate the association between cataracts and the concurrent presence of OSA and DM.

\section{METHODS}

\section{Subjects}

The study population was participants involved in the community-based cohort of the Korean Genome and Epidemiology Study (KoGES). The KoGES began in 2001 with 5020 Koreans (2523 men and 2497 women) aged 40-69 years. Detailed information on the study design and aims is available elsewhere $[16,17]$. Participants received comprehensive health examinations and questionnaire-based interviews during every follow-up visit. We used cataract data for all participants from 2009 to 2010, as well as sleep data acquired between 2009 and 2011 for OSA. We included participants who had undergone both examinations for cataracts and polysomnography (PSG). After excluding cases with missing data, we included the data from 699 individuals (286 men and 413 women) for analysis. All participants provided informed consent, and the study was approved by the $\mathrm{Hu}-$ man Subjects Review Committee at Korea University Ansan Hospital (2006AS0045).

\section{Diagnosis of Cataracts}

The Lens Opacities Classification System III is a standard system used for grading cataracts. The classification evaluates four features: nuclear opalescence (NO), nuclear color (NC), cortical cataract $(\mathrm{C})$, and posterior subcapsular cataract $(\mathrm{P})$. NO and $\mathrm{NC}$ are graded on a decimal scale from 1 to 6 , based on a set of six standardized photographs. $\mathrm{C}$ and $\mathrm{P}$ are graded on a decimal scale from 1 to 5 . The cataract group included participants with minimal changes $(1<\mathrm{NO}, 1<\mathrm{NC}, 1<\mathrm{C}, 1<\mathrm{P})$.

\section{PSG}

Unattended home PSG was done using a portable sleep monitoring device (Embletta X-100; Embla Systems, San Carlos, CA, USA), which consisted of a one-channel electroencephalogram, electrooculogram, chin electromyogram, a pressure transducer air-flow sensor, thoracic and abdominal respiratory movements sensor, electrocardiogram, and pulse oximetry. Using the American Academy of Sleep Medicine scoring manual for respiratory events [18], we defined apnea as a clear decrease in airflow of at least $90 \%$ from a previous baseline for a period of $10 \mathrm{sec}-$ onds, and hypopnea as a reduction in the oronasal flow $\geq 30 \%$ from a previous baseline, associated with at least $4 \%$ oxygen desaturation on pulse oximetry. We judged that OSA was present if the apnea-hypopnea index (AHI) was greater than 5 . We defined mild and moderate to severe OSA as $5 \leq \mathrm{AHI}<15$ and $\mathrm{AHI} \geq 15$, respectively.

\section{Questionnaire on Lifestyle, Anthropometric Measurements, and Definition of Disease}

We calculated body mass index (BMI) by dividing weight in kilograms by height in meters squared. We obtained information on physical activity (metabolic equivalent per hour daily, MET), alcohol intake (g/day), and smoking status (never, former, current) by questionnaires at each participant's visit. We defined hypertension (HTN) as systolic/diastolic blood pressure $\geq 140 / 90$ $\mathrm{mm} \mathrm{Hg}$ or a patient taking antihypertensive drugs. We defined $\mathrm{DM}$ as high fasting blood glucose of greater than $100 \mathrm{mg} / \mathrm{dL}$ or the use of antihyperglycemic medications.

\section{Statistical Analysis}

The data are expressed as means \pm standard deviation. We assessed the significance of the means by using one-way analysis of variance for continuous variables and a chi-squared test for categorical variables. We conducted univariate and multivariate logistic regression analyses to estimate the odds ratio (OR) of cataracts in relation to DM and OSA severity and reported it with a 95\% confidence interval (CI). The potential confounding variables included in the multivariate models for DM were age, sex, BMI, the presence of HTN, alcohol intake (g/day), smoking sta- 
tus (never, former, or current), physical activity (MET), and OSA severity (normal, mild, and moderate to severe). For OSA in the multivariate models, the potential confounding variables were the same as those for the DM models, except that OSA severity was used instead of the presence of DM. An interaction model containing only two variables and their interaction used the logistic procedure based on the Wald test to fit a two-way logit.

\section{RESULTS}

\section{Characteristics of Study Samples}

The 699 participants who underwent PSG and cataract diag- noses were included in the analysis. The general characteristics of the participants summarized across the severity group of OSA are shown in Table 1. The OSA patients were significantly older and more obese than the normal group. In addition, there were significant differences in alcohol intake, sex distribution, and the presence of diabetes, HTN, and smoking status. Physical activity did not differ across the severity group.

\section{Prevalence of Cataracts According to DM and OSA Severity Groups}

The prevalence of cataracts gradually increased as OSA worsened (Fig. 1A). The prevalence of cataracts was higher in patients with DM than in normal subjects (Fig. 1B).

Table 1. Characteristics of OSA group

\begin{tabular}{|c|c|c|c|c|}
\hline \multirow{2}{*}{ Variables } & \multicolumn{3}{|c|}{ OSA group } & \multirow{2}{*}{ p-value } \\
\hline & Normal $(\mathrm{n}=399)$ & Mild $(\mathrm{n}=219)$ & Moderate/severe $(\mathrm{n}=81)$ & \\
\hline Age (years) & $56.7 \pm 6.4$ & $60.3 \pm 7.6$ & $61.7 \pm 7.8$ & $<0.01$ \\
\hline Body mass index $\left(\mathrm{kg} / \mathrm{m}^{2}\right)$ & $23.9 \pm 2.6$ & $25.6 \pm 2.8$ & $26.2 \pm 3.5$ & $<0.01$ \\
\hline Physical activity* & $183.8 \pm 218.0$ & $221.7 \pm 311.3$ & $244.6 \pm 296.1$ & 0.07 \\
\hline Alcohol (g/day) & $4.0 \pm 10.5$ & $7.3 \pm 15.3$ & $10.3 \pm 19.2$ & $<0.01$ \\
\hline Sex & & & & $<0.01$ \\
\hline Men & $137(34.3)$ & $104(47.5)$ & $45(55.6)$ & \\
\hline Women & $262(65.7)$ & $115(52.5)$ & $36(44.4)$ & \\
\hline Diabetes & & & & $<0.01$ \\
\hline No & $341(85.5)$ & $160(73.1)$ & $57(70.4)$ & \\
\hline Yes & $58(14.5)$ & $59(26.9)$ & $24(29.6)$ & \\
\hline Hypertension & & & & $<0.01$ \\
\hline No & $303(75.9)$ & $127(58.0)$ & $36(44.4)$ & \\
\hline Yes & $96(24.1)$ & $92(42.0)$ & $45(55.6)$ & \\
\hline Smoking & & & & $<0.01$ \\
\hline Never & $300(75.2)$ & $135(61.6)$ & $46(56.8)$ & \\
\hline Former & $69(17.3)$ & $65(29.7)$ & $28(34.6)$ & \\
\hline Current & $30(7.5)$ & $19(8.7)$ & $7(8.6)$ & \\
\hline
\end{tabular}

Values are mean \pm standard deviation or $\mathrm{n}(\%)$.

*Average daily metabolic equivalents per hour.

OSA: obstructive sleep apnea.
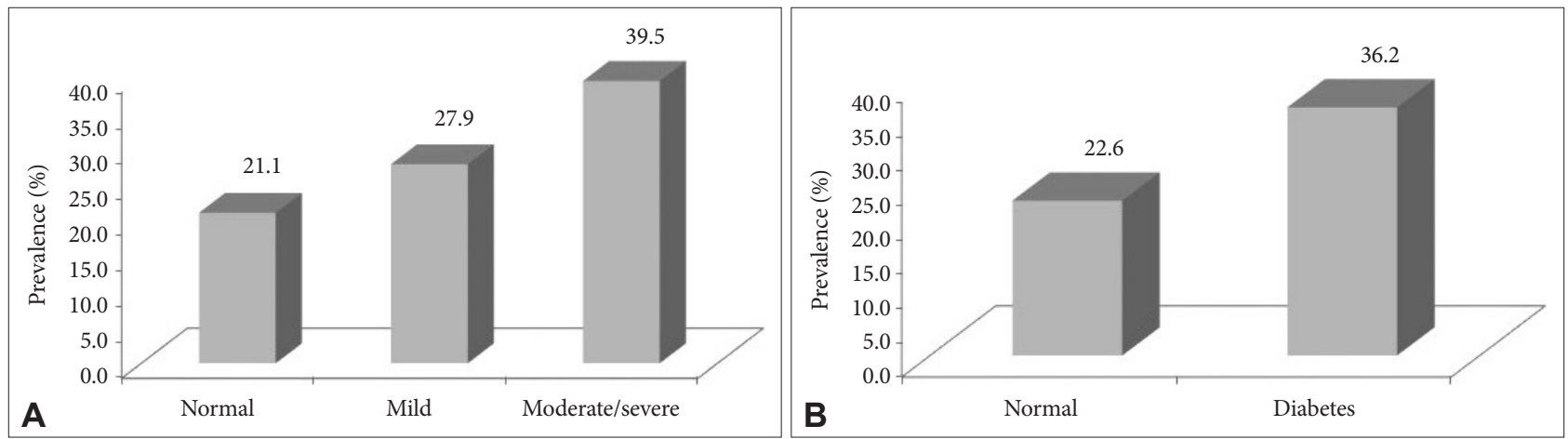

Fig. 1. Prevalence of cataracts according to the OSA severity and the presence of diabetes. A: Prevalence of cataracts in normal, mild, and moderate to severe OSA. B: Prevalence of cataracts in normal and diabetes patients. OSA: obstructive sleep apnea. 
Table 2. ORs of cataracts according to diabetes and OSA

\begin{tabular}{lccccc}
\hline & \multicolumn{2}{c}{ Unadjusted } & & \multicolumn{2}{c}{ Adjusted } \\
\cline { 2 - 3 } \cline { 5 - 6 } Diabetes & OR (95\% CI $)$ & p-value & & OR $(95 \%$ CI $)$ & p-value \\
Normal & Reference & & References \\
Diabetes & $1.94(1.31-2.89)$ & $<0.01$ & & $1.60(1.00-2.56)$ & $0.05^{*}$ \\
OSA & & & & \\
Normal & Reference & & Reference \\
Mild & $1.45(0.99-2.12)$ & 0.06 & $0.96(0.61-1.52)$ & $0.87^{\dagger}$ \\
Moderate/ & $2.45(1.48-4.06)$ & $<0.01$ & & $1.54(0.83-2.83)$ & $0.17^{\dagger}$ \\
severe & & & & \\
\hline
\end{tabular}

*Data were adjusted for age, sex, BMI, alcohol, smoking status, physical activity, the presence of HTN, and OSA groups. ${ }^{\circ}$ Data were adjusted for age, sex, BMI, HTN, alcohol, smoking status, physical activity, and the presence of diabetes and HTN.

OSA: obstructive sleep apnea, CI: confidence interval, OR: odds ratio, BMI: body mass index, HTN: hypertension.

Table 3. ORs of cataracts for the joint effects of diabetes and OSA

\begin{tabular}{|c|c|c|c|c|c|}
\hline \multirow{2}{*}{ Diabetes } & \multirow{2}{*}{ OSA } & \multicolumn{2}{|c|}{ Unadjusted } & \multicolumn{2}{|c|}{ Adjusted* } \\
\hline & & OR $(95 \% \mathrm{CI})$ & p-value & OR (95\% CI) & p-value \\
\hline Normal & Normal & \multicolumn{2}{|c|}{ Reference } & \multicolumn{2}{|c|}{ Reference } \\
\hline Diabetes & & $\begin{array}{c}1.53 \\
(0.81-2.88)\end{array}$ & 0.19 & $\begin{array}{c}1.12 \\
(0.54-2.30)\end{array}$ & 0.77 \\
\hline Normal & Mild & $\begin{array}{c}1.34 \\
(0.86-2.09)\end{array}$ & 0.20 & $\begin{array}{c}0.89 \\
(0.53-1.50)\end{array}$ & 0.65 \\
\hline Diabetes & & $\begin{array}{c}2.22 \\
(1.22-4.02)\end{array}$ & 0.01 & $\begin{array}{c}1.47 \\
(0.71-3.03)\end{array}$ & 0.30 \\
\hline Normal & $\begin{array}{l}\text { Moderate/ } \\
\text { severe }\end{array}$ & $\begin{array}{c}1.85 \\
(1.00-3.44)\end{array}$ & 0.05 & $\begin{array}{c}1.10 \\
(0.54-2.28)\end{array}$ & 0.79 \\
\hline Diabetes & & $\begin{array}{c}5.62 \\
(2.39-13.20)\end{array}$ & $<0.01$ & $\begin{array}{c}4.15 \\
(1.47-11.75)\end{array}$ & 0.01 \\
\hline
\end{tabular}

*Data were adjusted for age, sex, body mass index, alcohol, smoking status, physical activity, and the presence of hypertension. OSA: obstructive sleep apnea, CI: confidence interval, OR: odds ratio.

\section{ORs of Cataracts According to DM and OSA}

To estimate the ORs for cataracts according to the presence of DM and the severity of OSA, we did a logistic regression analysis (Table 2). In the crude analysis, the OR for cataracts in the presence of DM was 1.94 (95\% CI, 1.31-2.89; p < 0.01) and 2.45 (95\% CI, 1.48-4.06; $\mathrm{p}<0.01)$ in the presence of moderate/severe OSA. In the multivariate analysis, however, the significances disappeared.

\section{Joint Effects of DM and OSA on Cataracts}

The joint analysis for the presence of DM and OSA severity in association with cataracts is shown in Table 3. Crude logistic regression analysis showed a dose-dependent relationship between DM and cataracts according to OSA severity. However, this trend disappeared in the multivariate analysis adjusted for

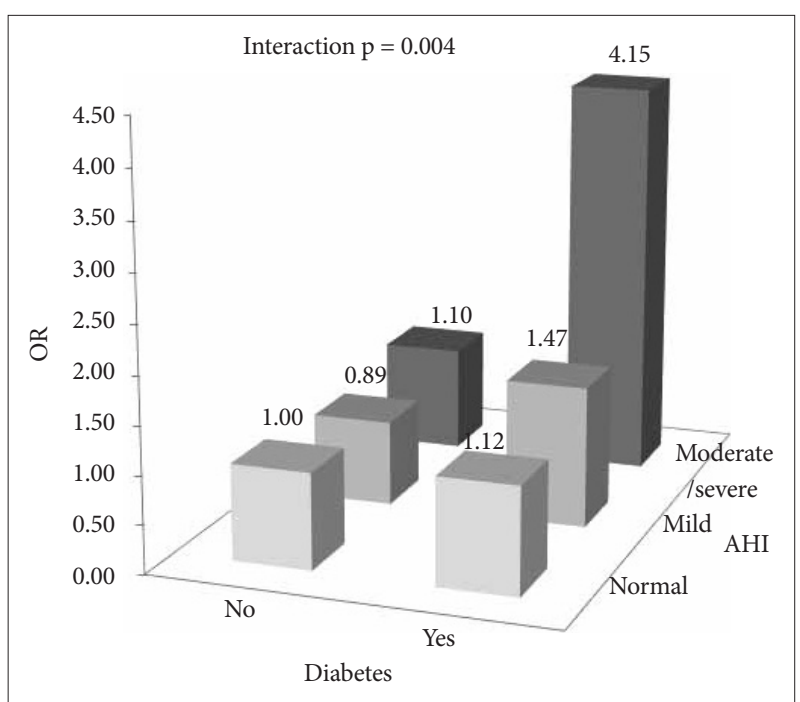

Fig. 2. ORs of cataracts in the joint analysis of the presence of diabetes and the severity of obstructive sleep apnea. OR: odds ratio, AHI: apnea-hypopnea index.

confounding variables. The significance remained only when DM coexisted with moderate to severe OSA. Next, we examined the interactions between the DM and OSA severity groups and the ORs for cataracts (Fig. 2) and found significant interactions ( $\mathrm{p}$ for interactions $=0.004$ ).

\section{DISCUSSION}

In this study we confirmed previous findings that the prevalence of cataracts was higher in diabetic patients than in normal subjects. We also found that the proportion of cataracts increased with increases in OSA severity. However, there was no significant association between DM and cataracts or between OSA and cataracts after we accounted for possible confounders. We observed a significant association only when DM coexisted with moderate to severe OSA.

Cataracts are very prevalent ocular disorders in diabetic patients. The risk of developing cataracts is up to five times higher in patients with DM, particularly at earlier ages [19-21]. Several mechanisms (e.g., polyol pathway, osmotic and oxidative stress, and autoimmunity) have been proposed for the development of diabetic cataracts.

Unlike previous findings that reported a significant association of cataracts and DM or OSA alone [15], we failed to observe any significant relationship between them in multivariate analysis. Possible explanations for this discrepancy follow. First, the studies did not consider DM (in a study on the association between OSA and cataracts) or OSA (in a study on the association between $\mathrm{DM}$ and cataracts) as confounding variables. Second, previous studies did not use a multivariate analysis taking OSA severity into account. Third, there were differences in study design, sample size, and race. Fourth, there can be some threshold level of 
specific factors or pathways to show cataract phenotype, and the individual presence of diabetes and OSA did not exceed the thresholds.

In addition, we wonder why this significant interaction effect was observed only in diabetic subjects with moderate to severe OSA. The lenses of diabetic patients are vulnerable to oxidative stress, one of the main pathological mechanisms induced by repeated oscillations of hypoxia and reoxygenation in OSA patients, because of their impaired antioxidant capacity [22]. According to a study that examined the oxidative stress levels according to the severity of OSA [23], the subjects who had an AHI > 15, corresponding to greater than moderate OSA, had reduced antioxidant capacities and greater systemic oxidative stress, implying that the oxidative stress in moderate to severe OSA was much higher than in mild OSA. In another study that prospectively investigated the association between NAION and OSA, 89\% of NAION patients had AHIs $\geq 15$. This study also suggested the importance of OSA severity in the development of ocular diseases. Thus, it is likely that only moderate to severe OSA with higher oxidative stress can lead to cataracts in patients with DM. This hypothesis can be proved by measuring actual levels of oxidative stress according to the severity of OSA.

The strength of our study was that it was a large populationbased study. Recent studies have reported possible associations between OSA and eye disorders, but many of these findings were in small case-control series or case reports, limiting the power of the evidence. The limitations of this study were, first, that it was not a prospective study but a cross-sectional study, so it is unclear whether OSA, DM, and cataracts are causally associated or merely share common risk factors. Second, we did not consider other OSA-related factors (e.g., sympathetic activity, hypercapnia, or inspiratory effort) that can play roles in ocular dysfunction. Thus, it is possible that this association was mediated by these additional factors.

In conclusion, this population-based study showed that the coexistence of moderate to severe OSA and DM was significantly associated with cataracts. Additional studies with prospective designs are needed to elucidate the causality among them. It is important for clinicians to understand the synergistic interaction between DM and OSA when evaluating individuals at risk for cataracts. In addition, OSA should be considered in the early detection of diabetic cataracts.

\section{Acknowledgments}

This research was supported by a fund (Grant 2009-E71002-00, 2010E71001-00, 2011-E71004-00) from the Korea Centers for Disease Control and Prevention.

\section{Conflicts of Interest}

The authors have no financial conflicts of interest.

\section{Authors' Contribution}

Conceptualization: Lee S, Yoon DW, Suh YW, Shin C. Data curation: Lee S, Yoon DW, Suh YW. Formal analysis: Lee S, Yoon DW. Funding acqui- sition: Shin C. Investigation: Lee S, Yoon DW, Suh YW, Shin C. Methodology: Lee S, Yoon DW, Shin C. Project administration: Shin C. Resources: Shin C. Supervision: Shin C. Visualization: Lee S. Writing-original draft: Lee S, Yoon DW, Kim J, Suh YW. Writing-review \& editing: Lee S, Yoon DW, Shin C.

\section{REFERENCES}

1. Young T, Palta M, Dempsey J, Skatrud J, Weber S, Badr S. The occurrence of sleep-disordered breathing among middle-aged adults. N Engl J Med 1993;328:1230-5.

2. Kim J, Hakim F, Kheirandish-Gozal L, Gozal D. Inflammatory pathways in children with insufficient or disordered sleep. Respir Physiol Neurobiol 2011;178:465-74.

3. Lavie L, Lavie P. Molecular mechanisms of cardiovascular disease in OSAHS: the oxidative stress link. Eur Respir J 2009;33:1467-84.

4. Shi Y, Liu P, Guan J, Lu Y, Su K. Association between glaucoma and obstructive sleep apnea syndrome: a meta-analysis and systematic review. PLoS One 2015;10:e0115625.

5. West SD, Turnbull C. Eye disorders associated with obstructive sleep apnoea. Curr Opin Pulm Med 2016;22:595-601.

6. Wu Y, Zhou LM, Lou H, Cheng JW, Wei RL. The association between obstructive sleep apnea and nonarteritic anterior ischemic optic neuropathy: a systematic review and meta-analysis. Curr Eye Res 2016;41: 987-92.

7. Leong WB, Jadhakhan F, Taheri S, Chen YF, Adab P, Thomas GN. Effect of obstructive sleep apnoea on diabetic retinopathy and maculopathy: a systematic review and meta-analysis. Diabet Med 2016;33:15868.

8. McNab AA. Reversal of floppy eyelid syndrome with treatment of obstructive sleep apnoea. Clin Exp Ophthalmol 2000;28:125-6.

9. Mojon DS, Mathis J, Zulauf M, Koerner F, Hess CW. Optic neuropathy associated with sleep apnea syndrome. Ophthalmology 1998;105:874-7.

10. Behbehani R, Mathews MK, Sergott RC, Savino PJ. Nonarteritic anterior ischemic optic neuropathy in patients with sleep apnea while being treated with continuous positive airway pressure. Am J Ophthalmol 2005;139:518-21.

11. Mitchell P, Cumming RG, Attebo K, Panchapakesan J. Prevalence of cataract in Australia: the Blue Mountains Eye Study. Ophthalmology 1997;104:581-8.

12. Chua J, Koh JY, Tan AG, Zhao W, Lamoureux E, Mitchell P, et al. Ancestry, socioeconomic status, and age-related cataract in Asians: the Singapore Epidemiology of Dye Diseases Study. Ophthalmology 2015; 122:2169-78.

13. Varma R, Torres M; Los Angeles Latino Eye Study Group. Prevalence of lens opacities in Latinos: the Los Angeles Latino Eye Study. Ophthalmology 2004;111:1449-56.

14. Liu YC, Wilkins M, Kim T, Malyugin B, Mehta JS. Cataracts. Lancet 2017;390:600-12.

15. Morsy NE, Amani BE, Magda AA, Nabil AJ, Pandi-Perumal SR, BaHammam AS, et al. Prevalence and predictors of ocular complications in obstructive sleep apnea patients: a cross-sectional case-control Study. Open Respir Med J 2019;13:19-30.

16. Kim J, In K, Kim J, You S, Kang K, Shim J, et al. Prevalence of sleepdisordered breathing in middle-aged Korean men and women. Am J Respir Crit Care Med 2004;170:1108-13.

17. Baik I, Kim J, Abbott RD, Joo S, Jung K, Lee S, et al. Association of snoring with chronic bronchitis. Arch Intern Med 2008;168:167-73.

18. Iber C. The AASM manual for the scoring of sleep and associated events: rules, terminology, and technical specification. 1th ed. Westchester, IL: American Academy of Sleep Medicine 2007.

19. Klein BE, Klein R, Lee KE. Diabetes, cardiovascular disease, selected cardiovascular disease risk factors, and the 5-year incidence of age-related cataract and progression of lens opacities: the Beaver Dam Eye Study. Am J Ophthalmol 1998;126:782-90. 
20. Klein BE, Klein R, Moss SE. Incidence of cataract surgery in the Wisconsin Epidemiologic Study of Diabetic Retinopathy. Am J Ophthalmol 1995;119:295-300.

21. Saxena S, Mitchell P, Rochtchina E. Five-year incidence of cataract in older persons with diabetes and pre-diabetes. Ophthalmic Epidemiol 2004;11:271-7.
22. Kiziltoprak H, Tekin K, Inanc M, Goker YS. Cataract in diabetes mellitus. World J Diabetes 2019;10:140-53.

23. Cekerevac I, Petrovic M, Lazic Z, Novkovic L, Cupurdija V. Severity of obstructive sleep apnea and oxidative stress. Eur Respir J 2015; 46: PA2324. 